\title{
Stefano Ragni, I viaggiatori musicali del Settecento Italiano: vol. IV; "Consuelo" di George Sand
}

\section{Annarosa Poli}

\section{(2) OpenEdition}

1 Journals

\section{Edizione digitale}

URL: https://journals.openedition.org/studifrancesi/39656

DOI: 10.4000/studifrancesi.39656

ISSN: 2421-5856

\section{Editore}

Rosenberg \& Sellier

\section{Edizione cartacea}

Data di pubblicazione: 1 décembre 2004

Paginazione: 395-397

ISSN: 0039-2944

\section{Notizia bibliografica digitale}

Annarosa Poli, «Stefano Ragni, I viaggiatori musicali del Settecento Italiano: vol. IV; "Consuelo" di George Sand», Studi Francesi [Online], 143 (XLVIII | II) | 2004, online dal 30 novembre 2015, consultato il 19 mai 2021. URL: http://journals.openedition.org/studifrancesi/39656 ; DOI: https://doi.org/10.4000/ studifrancesi.39656

Questo documento è stato generato automaticamente il 19 mai 2021.

\section{(c) (1)}

Studi Francesi è distribuita con Licenza Creative Commons Attribuzione - Non commerciale - Non opere derivate 4.0 Internazionale. 


\title{
Stefano Ragni, I viaggiatori musicali del Settecento Italiano: vol. IV; "Consuelo" di George Sand
}

\author{
Annarosa Poli
}

\section{NOTIZIA}

STEFANO RAGNI, I viaggiatori musicali del Settecento Italiano: vol. IV; “Consuelo" di George Sand. Perugia, Guerra edizioni, 2003, pp. 251.

1 Un libro che anticipa di pochi mesi l'apertura delle celebrazioni ufficiali per il bicentenario della nascita di George Sand. Un testo sulla musica che coglie soltanto alcuni degli estesi e ben temperati interessi che per tutta la vita legarono la signora di Nohant all'arte dei suoni. Approfondito, ben connotato fin nei minimi particolari il rapporto fra la Sand e Chopin, il più illustre fra gli artisti che fanno da corolla al genio sandiano. Ma la discendente di Maurizio di Sassonia aveva la musica nel sangue e quando approderà al sodalizio con Chopin aveva già costellato i suoi romanzi di estese e ben motivate presenze musicali.

Quando scrive Consuelo, tra il 1842 e il '44, siamo già in una fase problematica del rapporto Aurore-Fréderic. L'artista polacco ispira certamente molti tratti panslavi del protagonista maschile del romanzo, il conte Albert di Rudolstadt, ma certamente non condivideva le aperture democratiche e populistiche che fanno da tesi alla sezione più meditatamente sociale del romanzo. Anche Liszt, alla lettura di Consuelo, vi trovò espresse in eccesso quelle fantasticherie che avevano animato tante sue conversazioni con l'allora giovane madame Dudevant, che lui, il pianista magiaro, ormai attratto nell'orbita di madame D'Agoult, aveva superate.

Romanzo in chiave musicale questa Consuelo ripercorre, modula ed enfatizza la vita di una creatura fantastica che porta questo nome, ignorando le sue stesse origini. Forse è spagnola, probabilmente è zingara. La troviamo, all'inizio del romanzo, a Venezia, nella 
scuola vocale di Porpora, a studiare il canto. Ne L'Histoire de ma vie George Sand parla a più riprese della sua educazione accurata, dei tanti insegnamenti musicali della nonna M. me Dupin de Francueil che rivive in Consuelo, in quanto l'eroina del romanzo appartiene al secolo di Marie-Aurore, il Settecento. È la grande musica veneziana e napoletana quella che percorre tutte le pagine del romanzo e Porpora, quale creatore di voci eccezionali, ne è la chiave. Porpora, maestro di Farinelli, di Caffarelli, di Porporino, ovvero i diamanti della corona di evirati che attraversò l'Europa di corte in corte, diffondendo quella lingua italiana di Metastasio che costituiva il livello medio di educazione di ogni aristocratico di rango.

4 Anche Consuelo per una infelice storia d'amore, è costretta a lasciare Venezia e il suo addio pare straziante e definitivo. Ma sulle strade del vecchio continente dell'Ancien Régime gli incontri e gli scontri saranno molteplici. Maria Teresa d'Austria, Metastasio e Franz Joseph Haydn appariranno nelle pagine di Consuelo rivestiti di un'incredibile vitalità, sottratti alla sempre misteriosa icasticità della storia per divenire figure palpitanti, piene di buone intenzioni che spesso sono foriere solo di disgrazie. Singolare la penna di George Sand quando sbeffeggia Metastasio mettendo in ridicolo la sua vellutata ipocrisia. Maria Teresa ha urgenze da massaia e fobie da beghina. Haydn, il musicista degli Estérhazy, il padre della sinfonia, è visto sotto l'ottica di una ridente giovinezza, difficilmente esplorabile da una penna che non sia quella furtiva di un romanziere.

5 Il lato tenebroso di Consuelo è costituito dai personaggi dei castelli boemi, i conti di Rudolstadt e in particolare da Albert, personaggio che oscilla tra catalessi, visioni e perdite di coscienza. Vive molte vite Albert, ma Consuelo è affascinata da quella di ultimo esponente della setta degli Hussiti, i portatori di un credo cristiano ed utopistico. George Sand ne abbraccia la causa perché li considera eroi perseguitati e martiri per amore di libertà. Per giunta Albert è anche massone e come esponente della confraternita illuministica, diffonde per tutta l'Europa i germi della libertà.

Ampie ricognizioni storiche e bibliografiche consentono al romanzo di rimanere credibile anche nei momenti in cui la musica diventa talmente invasiva da sfiorare $\mathrm{i}$ contorni dell'assurdo: Albert suona nella sua caverna il violino Stradivari e ne trae melodie inaudite e, francamente, almeno da come le descrive la Sand, inaudibili. La potenza seduttrice della voce di Consuelo, l'unico tramite che ricongiunge Albert alla realtà, si ispira a quella reale di Pauline Viardot, la figlia di Manuel Garcia, il primo Almaviva rossiniano, la sorella cadetta della mitica Malibran. È lei la vera protagonista del romanzo, è sua la voce che esce dalla gola di Consuelo, sue sono le nozioni di tecnica troppo preziosamente distribuite in mille pagine, per essere esclusivo appannaggio di George Sand.

7 Purezza, sete di libertà, desiderio di fare del bene, necessità di conferire al teatro e al melodramma la dignità di un messaggio sociale e politico provengono dalle riflessioni musicali dei sansimoniani. La repubblicana Sand le fa sue e le mette in bocca a Consuelo.

8 Davanti alla cantante veneziana si ergerà un muro invalicabile: la diffidenza, l'astio $\mathrm{e}$ poi l'odio di Federico di Prussia, anche lui trasformato in personaggio di romanzo.

9 Presenza più che verosimile, poiché il despota del Brandeburgo era un accanito melomane, suonava il flauto e ascoltava l'opera italiana nei suoi teatri di corte, a Berlino e a Postdam. Consuelo ne scatena l'ira e ne subisce le conseguenze, non senza 
essere tutelata e protetta da un manipolo di musicisti federiciani che rispondono ai nomi storici dei fratelli Benda, di Graun, di Quantz e degli Starnitz.

Quando Consuelo si trasforma in Contessa di Rudolstadt è aperto conflitto tra assolutismo e illuminismo: la musica diventa campo d'azione anche quando Consuelo viene accolta nella setta di libertari e diventa lei stesso veicolo di democrazia. La musica è ora sublimata in istanza di accesso a un mondo di valori superiori, da mundana la musica diventa divina e addita le strade della Sublime Povertà, dell'uguaglianza, della fraternità. La rivoluzione francese è alle porte, ma gli Illuminati sono perseguitati, colpiti e dispersi, Consuelo perde la voce, diventa madre, si fa carico di Albert, ormai diventato un vaticinante Ermete Trismegisto. La mesta carovana si perde sui sentieri di Boemia e va verso la morte, o, forse, conclude George Sand, verso il trionfo.

11 Senza sfiorare l'amatissimo Rossini che già da Rose et Blanche era oggetto della sua predilezione, glissando sul Mozart massone ma antiliberale, la Sand ha inserito nel suo dittico tutto quel che alla sua epoca si poteva sapere sulla musica del Settecento italiano. Sorprende l'esattezza di certe intuizioni, ad esempio quella riesumazione dei Salmi di Benedetto Marcello, punta espressiva della prima parte parte del romanzo, ma presenti nel repertorio della Viardot.

12 Fanno capolino tra le pagine musicisti che solo l'esegesi del secondo Novecento avrebbe riportato in vita in indispensabili edizioni critiche: Holzbauer, Reuter, Durante, Lotti. La Sand li aveva scovati tra le pieghe dei suoi appunti e vi aveva alitato un soffio di vita.

Questo libro, scritto in uno stile vivace e animato, è indirizzato principalmente a un pubblico italiano (anche le citazioni di autori stranieri sono tutte tradotte così come $\mathrm{i}$ titoli delle opere).

14 Precisa e aggiornata la bibliografia. Ricca la documentazione erudita che sfrutta fonti di non facile riperimento e fa del volume un prezioso strumento per meglio conoscere l'atteggiamento di George Sand di fronte alla musica italiana.

Nella prossima edizione ci auguriamo vengano eliminati alcuni errori di stampa che figurano in particolare nelle note. 